\title{
Remerciements aux lecteurs arbitres
}

Natures Sciences Sociétés tient à remercier l'ensemble des personnes, dont les noms suivent, qui ont accepté avec dévouement et déontologie d'évaluer les manuscrits soumis à la revue en 2019. Ils ont éclairé la prise de décision du comité de rédaction et ont ainsi contribué par leurs critiques exigeantes à la sélection des manuscrits soumis et à la qualité des articles acceptés pour publication dans la revue.
Ancey Véronique
Artaud Hélène
Bahers Jean-Baptiste
Ballet Jérôme
Bergé Jean-Sylvestre
Bertrand François
Brédif Hervé
Bretesché Sophie
Carnoye Leslie
Cartier Stéphane
Chateauraynaud Francis
Colletis Gabriel
Décamps Henri
Dégremont Marlène
Delhoum Catherine
Desfontaines Hélène
Doré Thierry
Dörries Matthias
Durand Mathieu
Durand Séverine
Dutreuil Sébastien
Fagnart Jean-François
Faure David
Fernandez-Manjarres Juan
Ferru Marie
Fortané Nicolas
Foyer Jean
Garin Patrice
Gauthier Mario
Ghiotti Stéphane
Ginelli Ludovic
Gobert Julie

Goetz Damien

Gourmelon Françoise

Grouiez Pascal

Guerbois Chloé

Guerrin Joana

Keck Frédéric

Koleva Petia

Labbouz Benoît

Laloë Francis

Le Bars Yves

Legris Martine

Magny Michel

Mazeaud Alice

Ménard François

Micoud André

Mitroi Veronica

Monsaingeon Baptiste

Moulin Anne-Marie

Mounet Jean-Pierre

Pirard Éric

Polge Étienne

Pottier Antonin

Raulet-Croset Nathalie

Reghezza Magali

Rodet Guy

Rüdinger Andreas

Servais Véronique

Steyaert Patrick

Timmermans Benoît

Vanuxem Sarah

Yans Johan

Zelem Marie-Christine 